\title{
Sindrome nefrosica steroido-resistente trattata con ACTH
}

\author{
P. Lorusso, I. Cipollini, A. Bottai, G. Barsotti
}

U.O. Nefrologia e Dialisi 2 Universitaria, DAI Endocrinologia e Rene, Azienda Ospedaliero-Universitaria

Pisana, Pisa

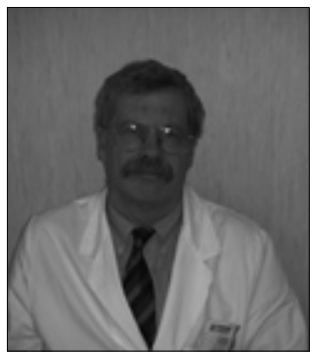

Paolo Lorusso

\section{Caso clinico}

Nel dicembre 2005 si presentava alla nostra osservazione una paziente di 17 anni a seguito di recente comparsa di edema periferico e aumento ponderale di oltre 5 $\mathrm{kg}$ nell'ultimo mese.

L'esame obiettivo all'ingresso era caratterizzato da edema improntabile periferico, pressione arteriosa di 130/80 $\mathrm{mmHg}$, peso corporeo di $59 \mathrm{~kg}$. Gli esami ematochimici e urinari effettuati all'ingresso evidenziavano: Creatininemia 0,7 mg/dL; Urea pl. $19 \mathrm{mg} / \mathrm{dL}$; Clearance creatinina $187 \mathrm{~mL} / \mathrm{min}$; Proteinemia totale 5,0 g/dL (Albuminemia 2,6 g/dL; alfa 1 globuline 3,8\%; alfa $223,7 \%$, Beta 12,1\%; Gamma 7,6\%); Colesterolemia totale $333 \mathrm{mg} /$ dL, Colesterolo HDL $58 \mathrm{mg} / \mathrm{dL}$; Colesterolo LDL 231 mg/dL; Trigliceridi 68 mg/dL; IgG 232 mg/dL; IgA 103 mg/dL; IgM 176 mg/dL; C3 108 mg/dL; C4 18 mg/dL. Proteinuria: $10 \mathrm{~g} / 24 \mathrm{~h}$. Esame del sedimento urinario: $144 \mathrm{GR} / \mathrm{mcl}, 6 \mathrm{~GB} / \mathrm{mcl}$.

Biopsia renale: nel preparato si trovano 7 glomeruli che presentano marcata proliferazione della matrice mesangiale ma senza evidenti alterazioni della membrana basale glomerulare. I lumi capillari si presentano pervi con frequenti dilatazioni e rare cellule infiammatorie nel loro contesto. Lumi tubulari talvolta ripieni di materiale proteico con estese aree di atrofia. Rari infiltrati interstiziali. Immunofluorescenza: $\operatorname{IgG}+++$ nastriforme diffuso, IgM ++ a zolle grossolane, catene leggere $\mathrm{K}$ +++ diffuse, lambda ++ diffuse.
Conclusioni: glomerulonefrite proliferativa mesangiale. La paziente è stata quindi trattata con terapia steroidea per tre mesi (prednisone $1.0 \mathrm{mg} / \mathrm{kg} / \mathrm{die}$ ) senza ottenere modificazioni della proteinuria. Inoltre alla paziente è stata prescritta la terapia comprendente Ramipril $5 \mathrm{mg}$, Lansoprazolo $30 \mathrm{mg}$, Atorvastatina $10 \mathrm{mg}$.

A distanza di un anno, persistendo il quadro di sindrome nefrosica conclamata, nel dicembre 2006 la paziente ha iniziato terapia con ACTH alla dose di $1 \mathrm{mg}$ i.m. alla settimana. Nel corso della terapia abbiamo osservato una lenta e progressiva riduzione della proteinuria che si è dimezzata dopo 4 mesi di terapia, passando da 10 a 4,2 $\mathrm{g} / 24$ ore. Successivamente la proteinuria ha continuato a ridursi fino a valori di $0,8 \mathrm{~g} / 24$ ore al termine del ciclo di 1 anno di terapia. Nel periodo successivo al trattamento si è verificata un'ulteriore riduzione della proteinuria, fino al raggiungimento di una remissione completa dopo 9 mesi dal termine del ciclo $(0,08 \mathrm{~g} / 24$ ore $)$. Tale remissione si è mantenuta fino a 2 anni dal termine del trattamento (Fig. 1).

Contemporaneamente alla riduzione della proteinuria si è assistito alla normalizzazione dei valori di albuminemia (Fig. 2). Contestualmente si è avuto il miglioramento del quadro lipidico (Fig. 3).

La funzione renale, espressa come clearance della creatinina, si è mantenuta nella norma durante e dopo il trattamento.

Il farmaco è stato ben tollerato e non si sono verificati effetti collaterali attribuibili alla terapia con ACTH.

La paziente ha sempre proseguito la terapia con ACE inibitore e statina, anch'essa sospesa dopo l'ottenimento della remissione completa della sindrome nefrosica. 


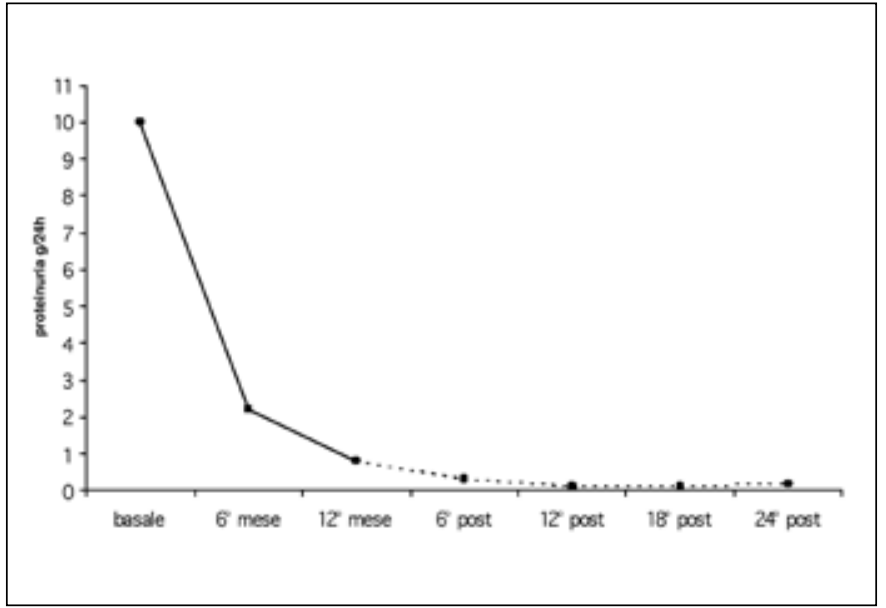

Fig. 1 - Andamento della proteinuria prima, durante e dopo trattamento con ACTH. Il periodo di trattamento è evidenziato con linea continua.

\section{Discussione}

La terapia con ormone adrenocorticotropo, o ACTH, già impiegata molti anni or sono nella sindrome nefrosica in sostituzione degli steroidi al fine di evitare l'insufficienza surrenalica, è stata recentemente rivalutata per l'efficacia in casi di sindrome nefrosica resistente a terapie convenzionali con cortisonici e immunosoppressori, in particolare nella glomerulonefrite membranosa (1-4). Infatti, un recente studio in pazienti con glomerulonefrite membranosa ha dimostrato che l'ACTH, alla dose di $1 \mathrm{mg} 2$ volte la settimana per un anno, aveva effetti sovrapponibili a quelli dello schema tradizionale con citostatici alternati a steroidi per 6 mesi (5).

Nel nostro caso abbiamo impiegato una dose di ACTH dimezzata rispetto a quella generalmente riportata in letteratura ottenendo una remissione completa della sindrome nefrosica. La proteinuria ha cominciato a ridursi dopo 4 mesi di trattamento ed è scomparsa dopo la fine del ciclo di anno di terapia con ACTH. La paziente non ha risposto agli steroidi ma ha risposto all'ACTH, attualmente non vi sono evidenze sperimentali che spieghino questo fenomeno. Si può ipotizzare che questo diverso comportamento sia mediato da modificazioni del metabolismo delle lipoproteine indotto dall'ACTH (2).

Nel nostro caso si è verificata una marcata riduzione della colesterolemia totale ed LDL (Fig. 3). Vi sono molte evidenze che supportano la responsabilità dei lipidi

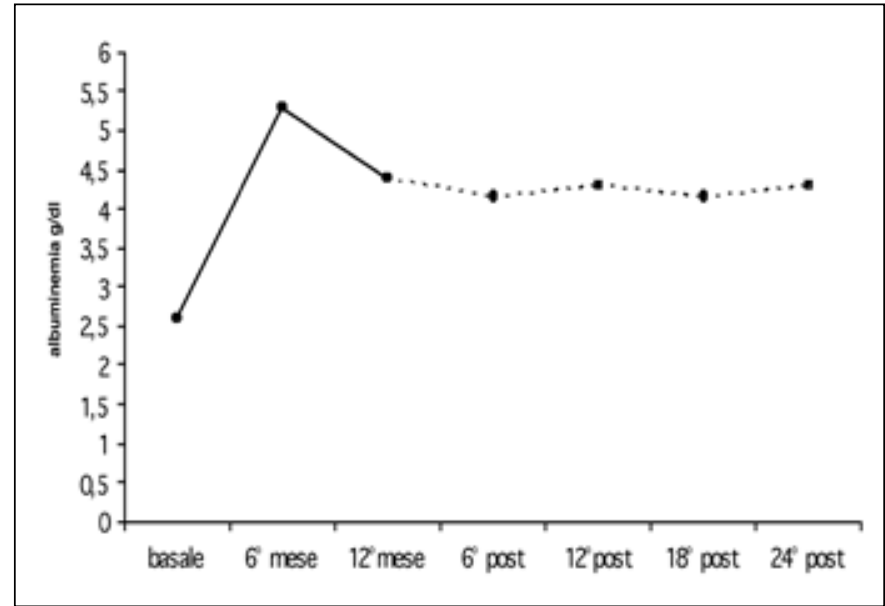

Fig. 2 - Andamento dell'albuminemia prima, durante e dopo trattamento con ACTH. Il periodo di trattamento è evidenziato con linea continua.

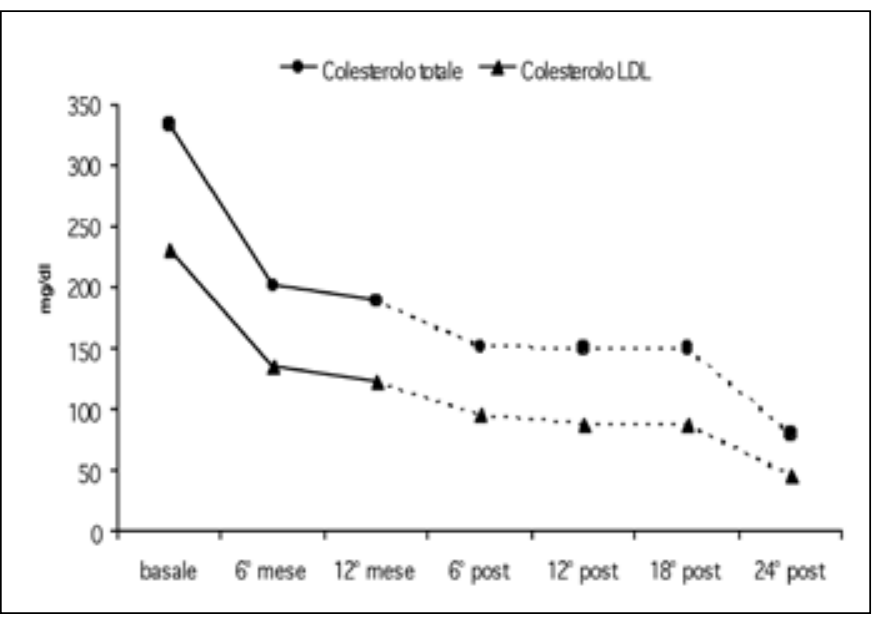

Fig. 3 - Andamento della colesterolemia totale e LDL prima, durante e dopo il trattamento con ACTH. Il periodo di trattamento è evidenziato con linea continua.

sul danno renale (6-8) e sulla sua progressione (9). Possiamo quindi pensare che la maggiore efficacia antiproteinurica dell'ACTH rispetto allo steroide possa essere messo in relazione anche al miglioramento dell'assetto lipidico, osservato anche da altri autori (2).

Come altra spiegazione a tale fenomeno possiamo ipotizzare un effetto più marcato e duraturo sul sistema immunitario.

La remissione della sindrome nefrosica riscontrata in questo caso può essere attribuita alla terapia o essere un'evoluzione spontanea della malattia stessa. Data la letteratura scientifica a tale riguardo (15) e in base alla nostra esperienza (10), possiamo 
affermare che la prima ipotesi sia quella più verosimile. $\grave{E}$ attualmente in corso un nostro studio che utilizza ACTH alla dose di $1 \mathrm{mg}$ i.m. la settimana per un anno in pazienti con proteinuria di grado nefrosico con diversi quadri istologici, resistenti a precedenti trattamenti con steroidi e immunosoppressori: al momento i primi risultati appaiono incoraggianti (10).

In conclusione, la nostra esperienza confor tata dai dati presenti in letteratura indica che la terapia con ACTH nella sindrome nefrosica ha i seguenti vantaggi rispetto alla terapia steroidea tradizionale:

- più marcato e duraturo effetto sulla proteinuria;

- minori effetti collaterali;

- assenza di insufficienza surrenalica post trattamento (11);

- riduzione della colesterolemia, noto fattore di rischio nelle nefropatie (6-9).

\section{Riassunto}

L'effetto antiproteinurico dell'ACTH è stato recentemente dimostrato nei pazienti con glomerulonefrite membranosa resistenti alla terapia convenzionale. Abbiamo praticato tale terapia in una paziente di 17 anni, giunta alla nostra osservazione con sindrome nefrosica (proteinuria 24h: $10 \mathrm{gr}$ ) e funzione renale normale. La paziente, con un quadro istologico di glomerulonefrite proliferativa mesangiale, era già stata sottoposta a terapia steroidea con prednisone $1 \mathrm{mg} / \mathrm{kg} /$ die per 3 mesi senza successo. Abbiamo impiegato dosi di ACTH più basse rispetto a quelle riportate in letteratura (1 mg i.m. alla settimana anziché $2 \mathrm{mg}$ ) per un anno, ottenendo una remissione completa della sindrome nefrosica: a distanza di 2 anni dalla fine della terapia la proteinuria è ancora assente. La funzione renale si è mantenuta stabile durante e dopo il trattamento, e non si sono verificati effetti collaterali legati al trattamento.

Parole chiave: Sindrome nefrosica, ACTH, Proteinuria, Glomerulonefrite proliferativa mesangiale

\section{Indirizzo dell'Autore:}

Paolo Lorusso, MD

U.O. Nefrologia e Dialisi 2 Universitaria

Azienda Ospedaliero-Universitaria Pisana

56126 Pisa, Italy

p.lorusso@ao-pisa.toscana.it

\section{TEST di VERIFICA - 2}

\section{1) Terapie alternative per la cura della glomerulo- nefrite membranosa:}
a) $\mathrm{ACTH}$
b) Rituximab
c) Ciclosporina
d) $a+b$

2) In quali condizioni possiamo decidere di trattare una glomerulonefrite con ACTH?

a) In qualsiasi glomerulonefrite appena diagnosticata

b) Nelle forme resistenti alla terapia convenzionale

c) Solo nelle forme primitive

d) $a+c$

\section{3) Vantaggi clinici della terapia con ACTH:}

a) Effetto più rapido sulla riduzione della proteinuria

b) Effetto più prolungato nel tempo sulla riduzione della proteinuria

c) Maggiore percentuale di remissione della sindrome nefrosica rispetto allo steroide

d) $a+b$

Le risposte corrette alle domande sono a pag. 25

\section{Bibliografia}

1. Coppo R. Non-steroidal and non cytotoxic therapies for nephritic syndrome. Nephrol Dial Transplant 2008; 23(6): 1793-96.

2. Berg AL, Nilsson-Ehle P, Arnadottir M. Benefical effects of ACTH on serum lipoprotein profile and glomerular function in patients with membranous nephropathy. Kidney Int 1999; 56: 1534-43.

3. Berg AL and Arnadottir M. ACTH-induced improvement in nephritic syndrome in patients with a variety of diagnoses. Nephrol Dial Transplant 2004; 19: 1305-07.

4. Rauen T, Michealis A, Fleoge J, Mertens PR. Case series of idiopathic membranous nephropathy with long-term beneficial effects of ACTH peptide 1-24. Clin Nephrol 2009; 71 (6): 637-42.

5. Ponticelli C, Passerini P, Salvadori M, et al. A Randomized 
Pilot Trial Comparing Methylprednisolone Plus a Cytotoxic Agent Versus Synthetic Adrenocorticotropic Hormone in Idiopatic Membranous Nephropathy. Am J Kid Dis 2006; 47 (2): 233-40.

6. Joles JA, Kunter U, Janssen U, et al. Early mechanisms of renal injury in hypercholesterolemic or hypertriglyceridemic rats. J Am Soc Nephrol 2000; 11(4): 669-83.

7. Attia DM, Ni ZN, Boer P, et al. Proteinuria is preceded by decreased nitric oxide synthesis and prevented by a NO donor in cholesterol-fed rats. Kidney Int 2002; 61: 1776-87.

8. Attia DM, Feron O, Goldschmeding R, et al. Hypercholesterolemia in rats induces podocytes stress and decreases renal cortical nitric oxide synthesis via an angio- tensin II type receptor-sensitive mechanism. J Am Soc Nephrol 2004; 15(4): 949-57.

9. Keane WF. Effect of lipids on glomerular injury and progression of renal disease. Verh K Acad Geneeskd Belg 1994; 56(2): 91-104.

10. Lorusso P, Cipollini I, Bottai A, Barsotti G. Effetto dell'ACTH sulla proteinuria nelle glomerulonefriti. Giornale Italiano di Nefrologia 2009; 26: S47, Settembre-Ottobre 2009, S32, Abstract.

11. Abeyagunawardena AS, Hindmarsh P, Trompeter RS. Adenocortical suppression increases the risk of relapse in nephritic syndrome. Arc Dis Child 2007; 92 (7): 585-8. 